\title{
Low Cost Amorphous Silicon Intrinsic Layer for Thin-Film Tandem Solar Cells
}

\author{
Ching-In Wu, ${ }^{1}$ Shoou-Jinn Chang, ${ }^{1}$ Kin-Tak Lam, ${ }^{2}$ Shuguang Li, ${ }^{3}$ and Sheng-Po Chang ${ }^{1}$ \\ ${ }^{1}$ Institute of Microelectronics \& Department of Electrical Engineering, Advanced Optoelectronic Technology Center, \\ Research Center for Energy Technology and Strategy, National Cheng Kung University, Tainan 70101, Taiwan \\ ${ }^{2}$ College of Physics and Information Engineering, Fuzhou University, Fuzhou, Fujian 350108, China \\ ${ }^{3}$ College of Science, China University of Petroleum (East China), Qingdao, Shandong 266555, China
}

Correspondence should be addressed to Shoou-Jinn Chang; changsj@mail.ncku.edu.tw

Received 19 September 2013; Accepted 2 October 2013

Academic Editor: Teen-Hang Meen

Copyright (C) 2013 Ching-In Wu et al. This is an open access article distributed under the Creative Commons Attribution License, which permits unrestricted use, distribution, and reproduction in any medium, provided the original work is properly cited.

\begin{abstract}
The authors propose a methodology to improve both the deposition rate and $\mathrm{SiH}_{4}$ consumption during the deposition of the amorphous silicon intrinsic layer of the a-Si/ $\mu \mathrm{c}-\mathrm{Si}$ tandem solar cells prepared on Gen 5 glass substrate. It was found that the most important issue is to find out the saturation point of deposition rate which guarantees saturated utilization of the sourcing gas. It was also found that amorphous silicon intrinsic layers with the same $k$ value will result in the same degradation of the fabricated modules. Furthermore, it was found that we could significantly reduce the production cost of the a-Si/ $\mu \mathrm{c}-\mathrm{Si}$ tandem solar cells prepared on Gen 5 glass substrate by fine-tuning the process parameters.
\end{abstract}

\section{Introduction}

In recent years, silicon-based thin-film solar cells have been studied extensively due to their potential benefits in low cost, high efficiency, and low pollution during production. It has been shown that these silicon-based thin-film solar cells are scalable for full-sized commercial production [1]. However, the fundamental challenge of silicon thin-film solar cells is light-induced degradation known as the StaeblerWronski Effect (SWE) [2]. There are currently two major types of silicon thin-film solar cells in the market. One is the amorphous silicon (a-Si) only module, which could achieve an approximately 7\% energy conversion efficiency with a degradation ratio of around $23 \%$. The other is the amorphous silicon tandem microcrystalline silicon (a-Si/ $\mu \mathrm{c}-$ $\mathrm{Si}$ ) tandem device, which could achieve more than $10 \%$ energy conversion efficiency with a smaller degradation ratio of around $15 \%$.

It is generally believed that increasing the ratio of hydrogen gas in the process of forming the intrinsic layers could improve the module stability. However, increasing hydrogen dilution ratio during the formation of the intrinsic layers suffers from two major drawbacks. First, hydrogen treatment could easily compromise p-layer interface in front of the intrinsic layer. Second, increasing the hydrogen dilution ratio of the total process gas also means decreasing the ratio of $\mathrm{SiH}_{4}$ used for forming the silicon film. This could result in a reduction in deposition rate. It has been reported previously that one can tune the distance of the electrodes to increase both the utilization of the depositing gas and the deposition rate [3]. However, it is necessary to adjust the hardware of the deposition system which depends strongly on the system used. It has also been reported that one can introduce triode to control the dissociation of reacting gas and the deposition rate [4]. However, adding one more electrode could increase the system cost and degrade the uniformity of the deposited layers. In this study, we propose a methodology to improve both the deposition rate and $\mathrm{SiH}_{4}$ consumption during the deposition of the amorphous silicon intrinsic layer of the a$\mathrm{Si} / \mu \mathrm{c}$-Si tandem solar cells prepared on Gen 5 glass substrate.

\section{Experiments}

In this work, amorphous silicon thin-film tandem solar cells with amorphous intrinsic layer prepared under different 
TABLE 1: A more detailed comparison between samples with two different hydrogen dilution ratios (i.e., 3 and 1).

\begin{tabular}{lcc}
\hline Intrinsic layer $(\mathrm{FR}=20.8 \mathrm{slm}, P=0.5 \mathrm{mbar}$, Dilu $=2.5)$ & Dilu $=3$ & Dilu $=1$ \\
\hline Depo. rate $(\mathrm{A} / \mathrm{sec})$ & 2 & 3.35 \\
$k$ & 0.619 & 0.618 \\
Uniformity & $9.95 \%$ & $15.72 \%$ \\
\hline
\end{tabular}

conditions were fabricated on Gen 5 glass substrate. The silicon active layer and the transparent conductive oxide (TCO), $\mathrm{ZnO}: \mathrm{B}$, and electrodes with a large area $(1300 \mathrm{~mm} \times$ $1100 \mathrm{~mm}$ ) in tandem were fabricated by very high frequency plasma-enhanced chemical vapor deposition (PECVD) with an excitation frequency of $40.68 \mathrm{MHz}$ and a low-pressure chemical vapor deposition (LPCVD) coater for anode and cathode, respectively. The current density-voltage $(J-V)$ characteristics of the produced module were then measured under a pulsed solar simulator system (Berger system). The system includes a solar simulator device (PSS $8 \mathrm{HS}$ ) under $500-1200 \mathrm{~W} / \mathrm{m}^{2}$ light intensity at $25^{\circ} \mathrm{C}$ and a load measuring device (PSL 8) with a power supply of $230 \mathrm{~V}-50 \mathrm{~Hz}, 1000$ Watt or $110 \mathrm{~V}-60 \mathrm{~Hz}$, and 1100 Watt. It should be noted that the $J-V$ measurement system used in this study was designed for fully automated module production lines. In some cases, we study a silicon single film by depositing the silicon single film directly on the front glass by PECVD. The full-sized single film sample was then measured by a large-area ellipsometer (J. A. Woollam, AccuMap-SE VI-1500). Deposition rate, spatial uniformity, refraction index $(n)$, and extinction coefficient $(k)$ were determined by fitting the optical raw data with a suitable optical model Cody-Lorentz.

We adjusted four major process parameters when forming the amorphous silicon intrinsic layer. These parameters include (1) total flow rate, $\mathrm{FR} \equiv \mathrm{SiH}_{4}$ flow + hydrogen flow, (2) pressure, $P$, (3) process power, $\mathrm{Pw}$, and (4) hydrogen dilution ratio, Dilu $\equiv\left[\mathrm{H}_{2}\right] /\left[\mathrm{SiH}_{4}\right]$. It is known that the total flow rate and pressure were mainly correlated with "radical residence time," while the process power and the hydrogen dilution ratio were mainly correlated with the "dissociation rate of radical" [5]. The basic approach that we took could be summarized as follows: first, we find the onset point of saturation for deposition rate (limited by radical dissociation) at a fixed "radical residence time." Second, we enhanced "residence time" to further raise the deposition rate.

After choosing potential fitting conditions, we used these specific parameters to form modules. To check the module stability, the fabricated modules were moved outdoors to soak in sunlight in Tainan, Taiwan. After soaking in enough sunlight according to the rules defined by the international electrotechnical commission (IEC) ( $>43 \mathrm{kw} / \mathrm{hr}$ ) in one cycle, the modules were moved inside to measure their efficiencies. If the measured modules were unstable in efficiency as defined by IEC ( $<2 \%$ degradation within three cycles), they were moved outdoors again for the next sunlight soaking cycle. The cycles were repeated until the efficiency became stable. Each module was then moved inside and put in room temperature in the dark for at least one day to prevent outdoor environmental influences before module power was measured.

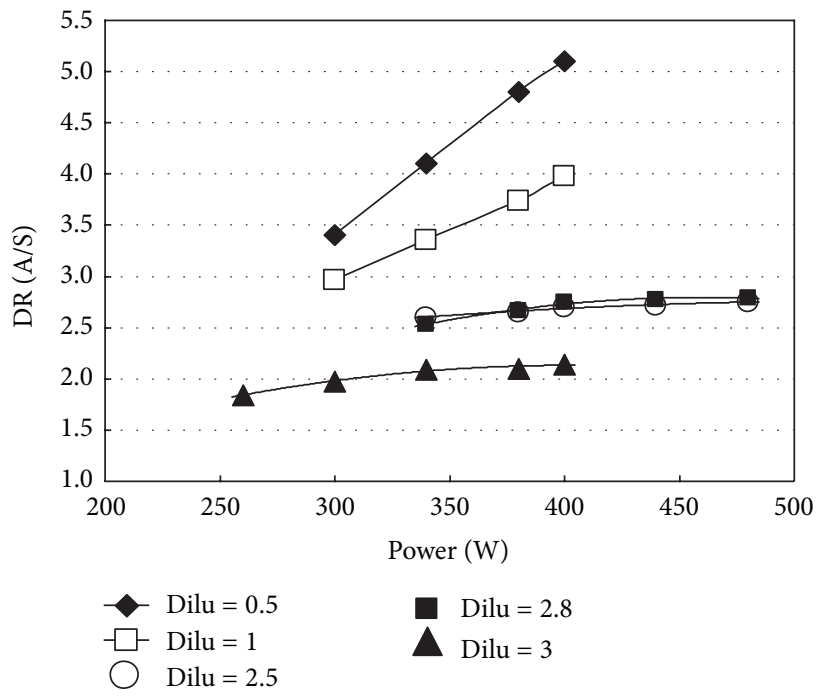

FIgURE 1: Deposition rate of the amorphous silicon intrinsic layer as a function of Dilu and Pw. FR and $P$ are kept at $20.8 \mathrm{slm}$ and 0.5 mbar, respectively.

\section{Results and Discussion}

An original sample was first prepared. For this sample, the amorphous silicon intrinsic layer was deposited using total flow rate $\mathrm{FR}=20.8 \mathrm{slm}$, pressure $P=0.5 \mathrm{mbar}$, process power $\mathrm{Pw}=340 \mathrm{Watt}$, and hydrogen dilution ratio, Dilu $=3$. It was found that the deposition rate was $2 \mathrm{~A} / \mathrm{sec}$ using these process parameters. We then studied deposition rate of the amorphous silicon intrinsic layer as a function of hydrogen dilution ratio, Dilu, and process power, $\mathrm{Pw}$, while keeping the total flow rate, FR, and pressure, $P$, at $20.8 \mathrm{slm}$ and 0.5 mbar, respectively. As shown in Figure 1, it can be seen that increasing the plasma power during deposition will lead to an increased deposition rate (DR). This should be attributed to the higher chance for $\mathrm{SiH}_{4}$ gas to dissociate. However, it was also found that such an enhancement was significantly suppressed as we increased the hydrogen dilution ratio. Table 1 summaries these results. With 340 Watt plasma power, $0.5 \mathrm{mbar}$ pressure, and $20.8 \mathrm{slm}$ total flow rate, it was found that we could increase the deposition rate from 2 to $3.35 \mathrm{~A} / \mathrm{sec}$, as we decreased the hydrogen dilution ratio from 3 to 1 . This suggests that we could easily achieve a $>40 \%$ enhancement in deposition rate of the amorphous silicon intrinsic layer by decreasing the hydrogen dilution ratio.

Figure 2 shows outdoor soaking results of the fabricated modulates with two different hydrogen dilution ratios (i.e., 3 and 1). It was found that lower hydrogen dilution ratio (Dilu = 1) could provide us a higher deposition rate of the amorphous 


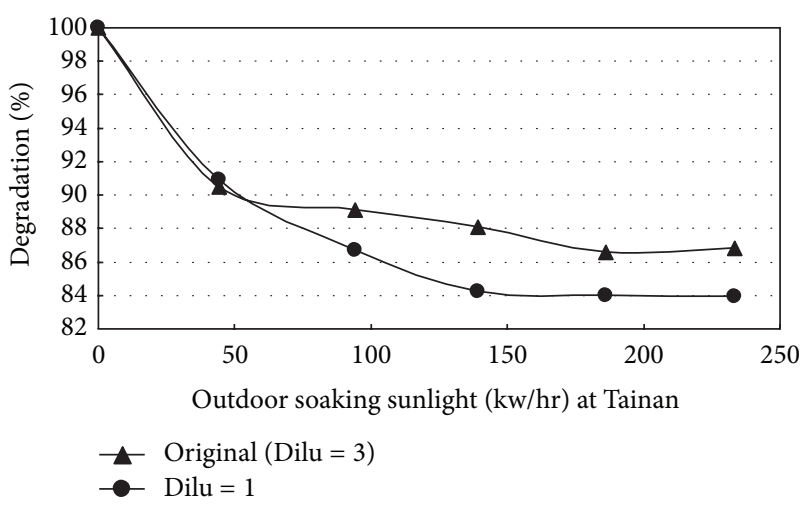

FIGURE 2: Outdoor soaking results of the fabricated modulates with two different hydrogen dilution ratios (i.e., 3 and 1).

intrinsic layer. However, it was found from Figure 2 that the degradation ratio became higher, as compared to the original sample with Dilu $=3$. Table 1 lists a more detailed comparison between these two samples. It can be seen that the dielectric constant, $k$, of the amorphous silicon intrinsic layer measured from these two samples was about the same. However, it was also found that thickness uniformity (i.e., 9.95\%) was much better for the sample prepared with Dilu $=3$, as compared to that prepared with Dilu $=1$ (i.e., $15.72 \%$ ). It is possible that the high degradation observed from the sample prepared with Dilu $=1$ was directly related to the thickness nonuniformity.

It has been shown previously that controlling the dissociation of reacting gas $\left(\mathrm{SiH}_{4}\right.$ and $\left.\mathrm{H}_{2}\right)$ is a key factor in achieving good stability of the amorphous silicon intrinsic layer $[6,7]$. It has also been shown that we could tune the process parameters to achieve similar crystal size of microcrystalline silicon layer [8]. Thus, we also tried to tune different gas resident time by changing total flow rate, deposition pressure, and plasma power so as to achieve different hydrogen dilution ratios during the deposition of the amorphous silicon intrinsic layer. As we modify different parameters in the experiment, this formula always remains constant:

$$
P V=N R T
$$

where $P$ is the pressure in the chamber, $V$ is the total chamber volume, $N$ is the number of moles, $R$ is the gas constant, and $T$ is the absolute temperature of the gas. It should be noted that $V, R$, and $T$ are constants for the same process chamber. In the experiment, we also kept the value of $P$ as a constant. Since only $\mathrm{SiH}_{4}$ and $\mathrm{H}_{2}$ were introduced into the process chamber, we could derive the following formula:

$$
P_{(\text {total })} V=P_{\left(\mathrm{SiH}_{4}\right)} V+P_{\left(\mathrm{H}_{2}\right)} V \text {. }
$$

With $P$ as a constant, we can simplify the formula to

$$
P_{(\text {total })}=P_{\left(\mathrm{SiH}_{4}\right)}+P_{\left(\mathrm{H}_{2}\right)} \text {. }
$$

By keeping $P_{\text {(total) }}$ constant, the partial pressure of $\mathrm{SiH}_{4}$ would have to decrease if we increase the hydrogen flow rate with a fixed flow rate of $\mathrm{SiH}_{4}$ gas. In other words, the resident time of

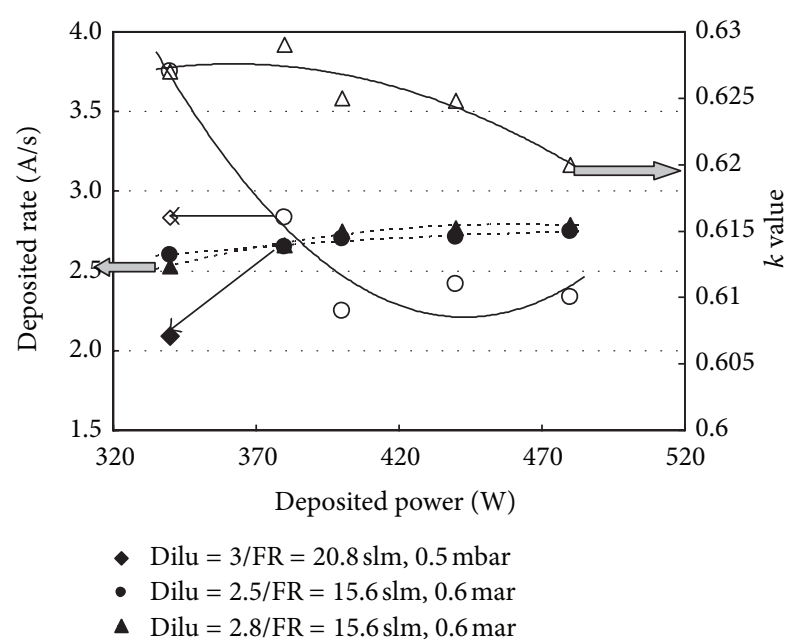

Figure 3: Deposition rate and $k$ values as functions of deposition power under three different conditions.

$\mathrm{SiH}_{4}$ has to decrease. The decreased partial pressure of $\mathrm{SiH}_{4}$ should result in a lower deposition rate of the silicon film. This could be used to explain why the deposition rate is inversely correlated with the dilution ratio of the hydrogen gas. For better usage of source gas, we should decrease the total flow rate and increase the resident time of the source gas in the chamber while maintaining the same pressure and the same ratio for $\mathrm{H}_{2}$ and $\mathrm{SiH}_{4}$.

To maximize the utilization of source gases, we should also optimize the deposition power. Figure 3 shows deposition rate and $k$ values as functions of deposition power under three different conditions. It can be seen that the deposition rate increased rapidly, reached a saturation point, and then increased slowly. The much slower increase in deposition rate after saturation point should be attributed to the saturation of dissociation. The saturation point in deposition rate also suggests the best composition of the specific ratio of source gas at the same dilution ratio, total flow rate, and pressure. It should be noted that undesired $\mathrm{SiH}_{4}$ polymer or dangling bond in intrinsic layer could occur easily in the unsaturated region. This could result in serious SWE and severe lightinduced degradation. Other than choosing the proper process parameters, we also need to check the $k$ value so as to know if the deposited amorphous silicon intrinsic layer exhibits the same absorption as that of the original sample. As shown in Figure 3, it can be seen that we could achieve a $k$ value matching that of the original sample (i.e., $k=0.618$ ) by choosing power $=380 \mathrm{~W}, \mathrm{FR}=15.6 \mathrm{slm}$, pressure $=0.6 \mathrm{mbar}$, and Dilu $=2.5$. With this matched $k$ value, we could use the deposited amorphous silicon intrinsic layer in our tandem module. As also shown in Figure 3, it was found that thickness uniformity of the sample prepared with these parameters was $11.12 \%$, which was reasonably good.

Figure 4 shows outdoor soaking results of the fabricated modulates with two kinds of amorphous silicon intrinsic layer after tuning (i.e., Dilu $=2.5$ and Dilu $=3$ ). These modules were used for the deep top-limited tandem device with the same $1600 \mathrm{~nm}$ thick $\mu \mathrm{c}$-Si device. For comparison, we only 
TABLE 2: The results of modulates fabricated with these three kinds of amorphous silicon intrinsic layer.

\begin{tabular}{lccc}
\hline Condition $(\mathrm{FR}, P, \mathrm{Pw}, \mathrm{Dilu})$ & $\mathrm{SiH}_{4}$ consumption & Tact time $(\mathrm{s})$ & Initial module power $(\mathrm{W})$ \\
\hline $20.8,0.5,380,1$ & $100.00 \%$ & 835 & 138.85 \\
$15.6,0.5,380,2.5$ & $102.60 \%$ & 1076 & 138.57 \\
$20.8,0.5,340,3$ (original) & $182.00 \%$ & 1400 & 138.75 \\
\hline
\end{tabular}

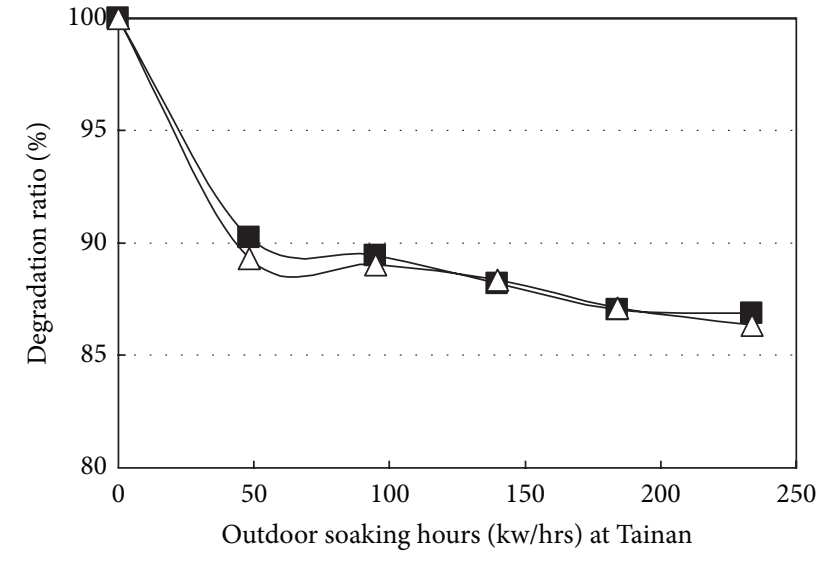

Dilu $=3,340 \mathrm{~W}, 0.5 \mathrm{mbar}, \mathrm{FR}=20.8 \mathrm{slm}$

$\neg$ Dilu $=2.5,380 \mathrm{~W}, 0.6 \mathrm{mbar}, \mathrm{FR}=15.6 \mathrm{slm}$

FIGURE 4: Outdoor soaking results of the fabricated modulates with two kinds of amorphous silicon intrinsic layer after tuning (i.e., Dilu $=2.5$ and Dilu $=3$ ).

changed the amorphous silicon intrinsic layer. The amount of sunlight was measured by SMA Sunny Web Box. It can be seen from Figure 4 that the degradation behaviors were similar for these two modules. After soaking for $240 \mathrm{~kW} / \mathrm{hr}$, it was found that these two modules both became stable with a degradation ratio of around 86\%, following the IEC61646 10.19.3 standard. We further fine-tuned the four parameters during the deposition of the amorphous silicon intrinsic layer. Other than the original sample, we also prepared two samples with two different process parameters during the deposition of the amorphous silicon intrinsic layer. One sample was prepared using total flow rate $\mathrm{FR}=20.8 \mathrm{slm}$, pressure $P=0.5$ mbar, process power $\mathrm{Pw}=380$ Watt, and hydrogen dilution ratio Dilu $=1$. The other sample was prepared using total flow rate $\mathrm{FR}=15.6 \mathrm{slm}$, pressure $P=0.5 \mathrm{mbar}$, process power $\mathrm{Pw}=380$ Watt, and hydrogen dilution ratio Dilu = 2.5. Table 2 lists the results of modulates fabricated with these three kinds of amorphous silicon intrinsic layer. It can be seen that the initial powers measured from these three modules were similar (i.e., $~ 139$ Watt). However, it was found that the amounts of $\mathrm{SiH}_{4}$ consumed during the deposition of the amorphous silicon intrinsic layers were very different. Assuming that the $\mathrm{SiH}_{4}$ consumption for the sample prepared with $\mathrm{FR}=20.8 \mathrm{slm}, P=0.5 \mathrm{mbar}, \mathrm{Pw}=380$ Watt, and Dilu $=1$ was $100 \%$, it was found that the $\mathrm{SiH}_{4}$ consumption for the sample prepared with $\mathrm{FR}=15.6 \mathrm{slm}, P=0.5 \mathrm{mbar}, \mathrm{Pw}$ $=380$ Watt, and Dilu $=2.5$ was around $102 \%$. Furthermore, it was found that the $\mathrm{SiH}_{4}$ consumption for the original sample prepared with $\mathrm{FR}=20.8 \mathrm{slm}, P=0.5 \mathrm{mbar}, \mathrm{Pw}=340$ Watt, and Dilu $=3$ was higher than $180 \%$. Furthermore, it was found that tact times observed from the sample prepared with FR = $20.8 \mathrm{slm}, P=0.5 \mathrm{mbar}, \mathrm{Pw}=380$ Watt, and Dilu $=1$ was $835 \mathrm{sec}$, while that observed from the sample prepared with $\mathrm{FR}=15.6 \mathrm{slm}, P=0.5 \mathrm{mbar}, \mathrm{Pw}=380$ Watt, and Dilu $=$ 2.5 was $1076 \mathrm{sec}$. In contrast, it was found that tact time observed from the original sample was as long as $1400 \mathrm{sec}$. Compared with the original sample, the much smaller $\mathrm{SiH}_{4}$ consumptions and the shorter tact times observed from the two fine-tuned samples suggest that we could significantly reduce the production cost of the a-Si/ $\mu \mathrm{c}-\mathrm{Si}$ tandem solar cells prepared on Gen 5 glass substrate.

\section{Conclusion}

In summary, we propose a methodology to improve both the deposition rate and $\mathrm{SiH}_{4}$ consumption during the deposition of the amorphous silicon intrinsic layer of the a-Si/ $\mu \mathrm{c}-\mathrm{Si}$ tandem solar cells prepared on Gen 5 glass substrate. It was found that the most important issue is to find out the saturation point of deposition rate which guarantee saturated utilization of the sourcing gas. It was also found that amorphous silicon intrinsic layers with the same $k$ value will result in the same degradation of the fabricated modules. Furthermore, it was found that we could significantly reduce the production cost of the a-Si/ $\mu \mathrm{c}-\mathrm{Si}$ tandem solar cells prepared on Gen 5 glass substrate by fine-tuning the process parameters.

\section{Acknowledgment}

The authors would like to thank the Bureau of Energy, Ministry of Economic Affairs of Taiwan, R.O.C. for the financial support under Contract no. 102-E0603.

\section{References}

[1] M. Kondo, T. Matsui, Y. Nasuno, H. Sonobe, and S. Shimizu, "Key issues for fabrication of high quality amorphous and microcrystalline silicon solar cells," Thin Solid Films, vol. 501, no. 1-2, pp. 243-246, 2006.

[2] D. L. Staebler and C. R. Wronski, "Reversible conductivity changes in discharge-produced amorphous Si," Applied Physics Letters, vol. 31, no. 4, pp. 292-294, 1977.

[3] Y. Nakano, S. Goya, T. Watanabe, N. Yamashita, and Y. Yonekura, "High-deposition-rate of microcrystalline silicon solar cell by using VHF PECVD," Thin Solid Films, vol. 506-507, pp. 33-37, 2006.

[4] S. Shimizu, H. Miyahara, M. Kondo, and A. Matsuda, "Fabrication of hydrogenated amorphous silicon films exibiting higher stability against light soaking," Journal of Non-Crystalline Solids, vol. 338-340, no. 1, pp. 47-50, 2004. 
[5] A. Gordijn, A. Pollet-Villard, and F. Finger, "At the limit of total silane gas utilization for preparation of high-quality microcrystalline silicon solar cells at high-rate plasma deposition," Applied Physics Letters, vol. 98, no. 21, Article ID 211501, 2011.

[6] A. Matsuda, M. Takai, T. Nishimoto, and M. Kondo, "Control of plasma chemistry for preparing highly stabilized amorphous silicon at high growth rate," Solar Energy Materials and Solar Cells, vol. 78, no. 1-4, pp. 3-26, 2003.

[7] T. Nishimoto, M. Takai, H. Miyahara, M. Kondo, and A. Matsuda, "Amorphous silicon solar cells deposited at high growth rate," Journal of Non-Crystalline Solids, vol. 299-302, no. 2, pp. 1116-1122, 2002.

[8] A. Matsuda, "Thin-film silicon-growth process and solar cell application," Japanese Journal of Applied Physics, vol. 43, no. 12, pp. 7909-7920, 2004. 

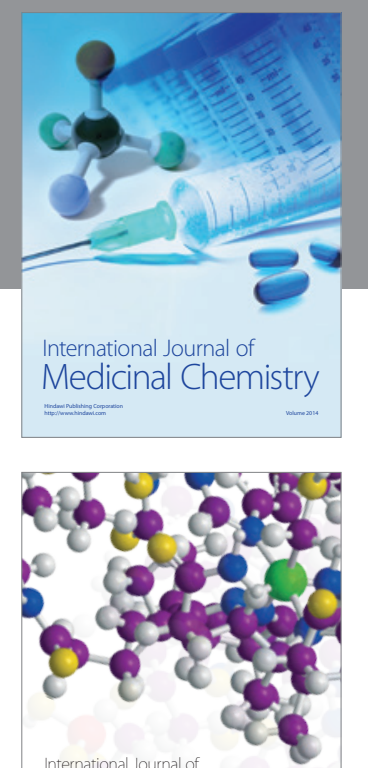

\section{Carbohydrate} Chemistry

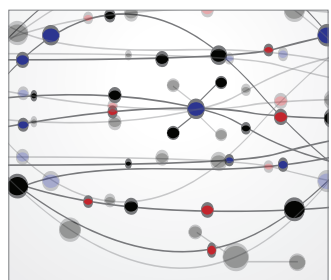

The Scientific World Journal
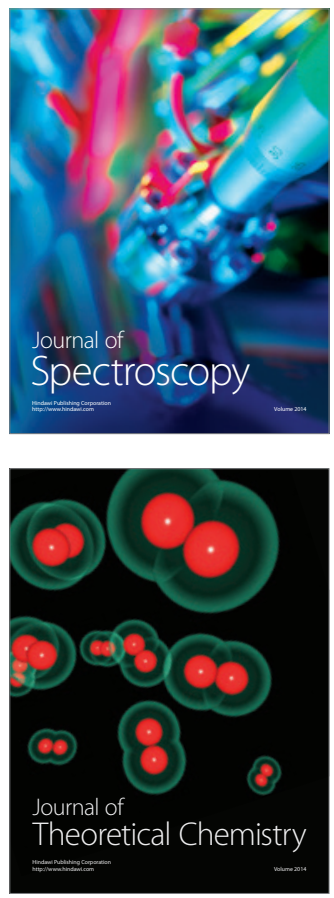
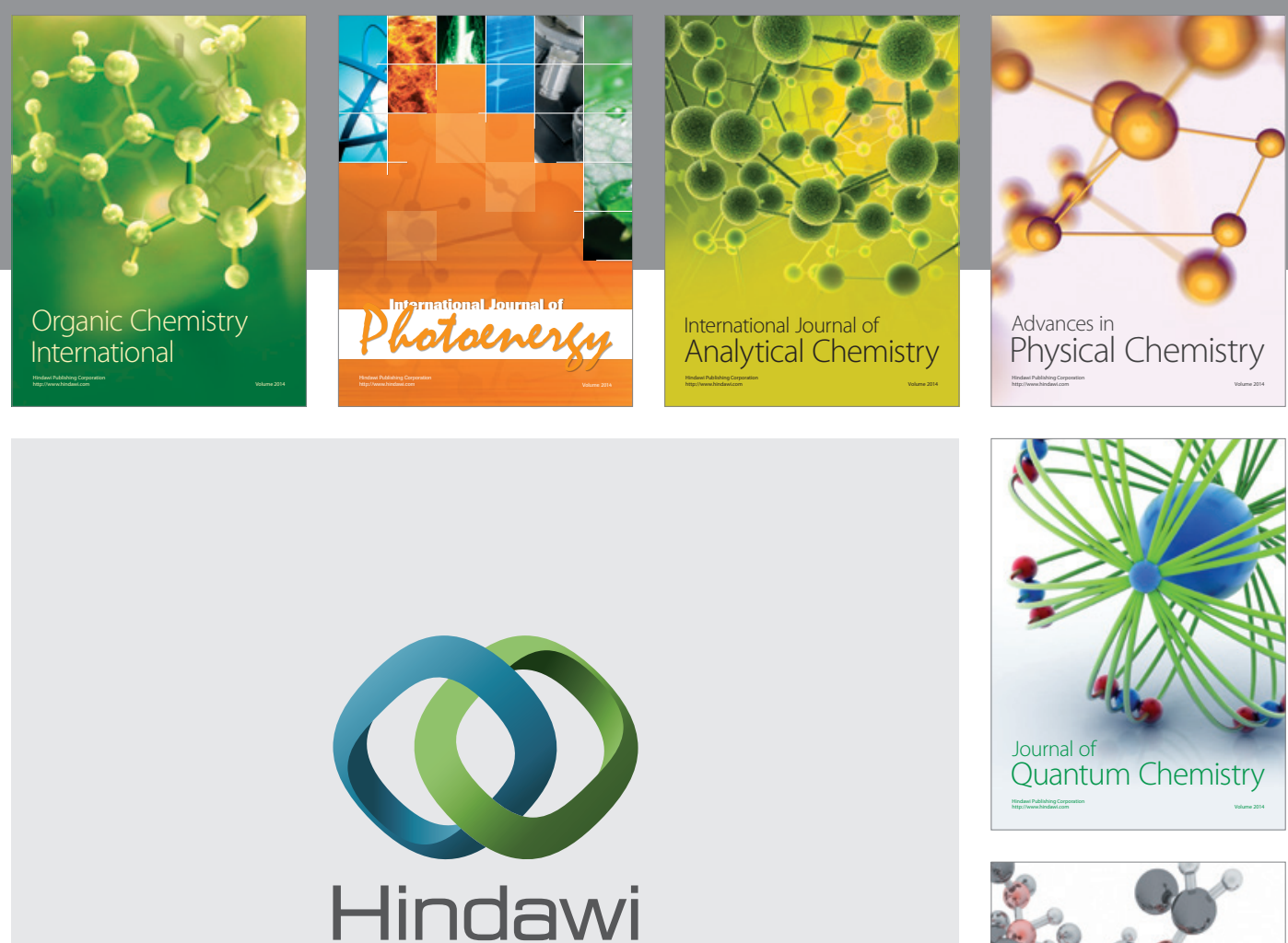

Submit your manuscripts at

http://www.hindawi.com

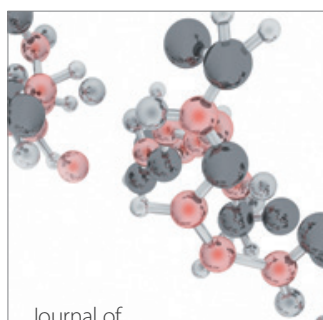

Analytical Methods

in Chemistry

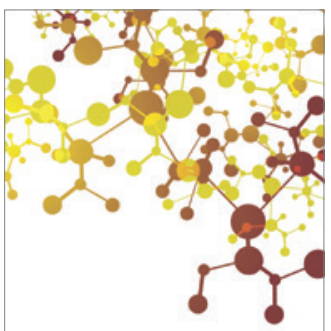

Journal of

Applied Chemistry

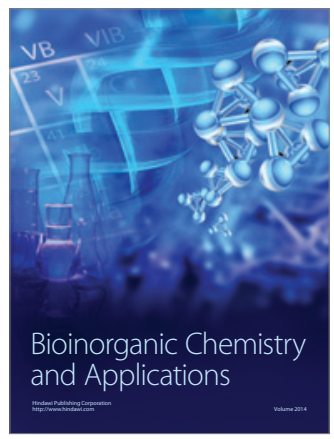

Inorganic Chemistry
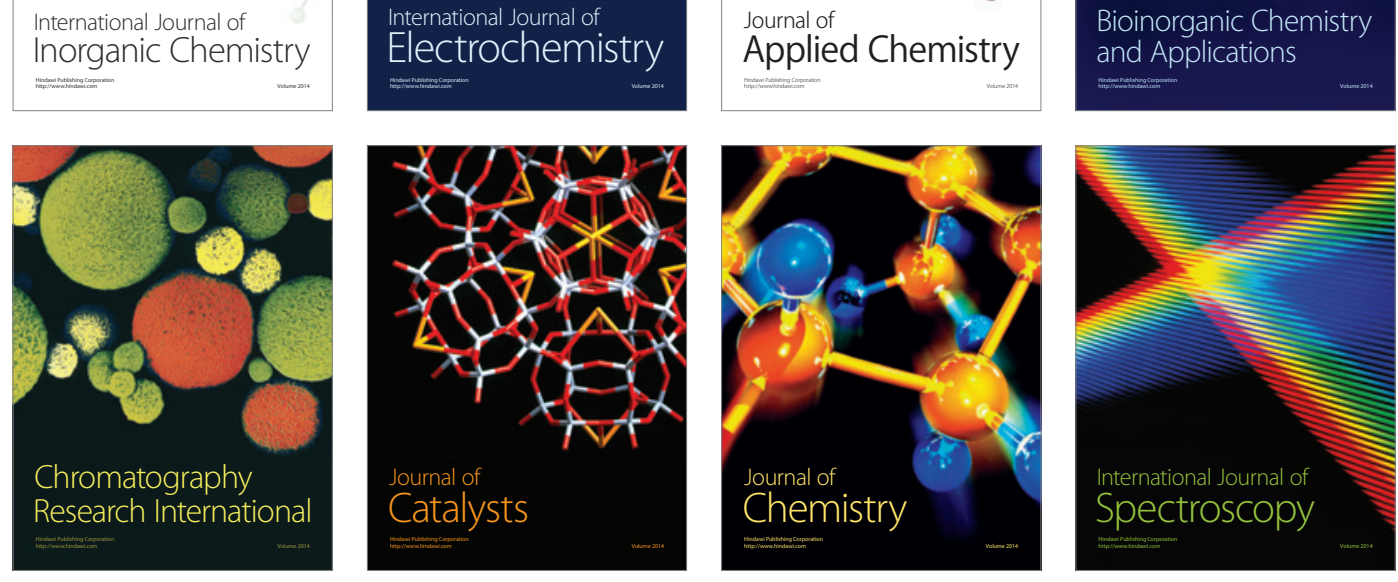